\title{
CANS Implementation using GSTEB Protocol in Wireless Sensor Network
}

\author{
R. Harish Kumar' ${ }^{1}$ M. Chandra Sekhar Rao ${ }^{2}$ \\ M. Tech, Department of CSE, MVGR College of Engineering, Vizianagaram, Andhra Pradesh, India ${ }^{1}$ \\ Assistant Professor, Department of CSE, MVGR College of Engineering, Vizianagaram, Andhra Pradesh, India ${ }^{2}$
}

\begin{abstract}
Wireless sensor network is the emerging area where we are largely depending on it, in our day to day life. The major application of the wireless sensor network is the emergency navigation service during the emergency situations, and the main goal of this service is to save the people from emergency situations by guiding them to reach a safe with small congestion and fewer detours. The existing systems are mainly focus on the safe exit to the people. But, they ignoring the problems like congestion and heavy detours caused due to people movement towards the exit on this kind of emergency situations. From this project, we propose a new existing algorithm named CANS which means Congestion Adaptive Small stretch and Emergency Navigation algorithm mainly used to control the congestion in emergency situations and guide the people to the safe locations within a short time. Because CANS doesn't need any location information. Without any location information, guiding the users for safe path is practically impossible. So, we propose the GSTEB protocol to guide the people in dangerous situations for a safe exit. By using CANS algorithm, when any emergency situation occurs, the sensors in the wireless sensor networks take the information on overall condition of the surroundings and location information of the all the users. And then the sensors in the wireless sensor networks talk with the mobile users about the better and near way to the exit or safe path. CANS execute the technique of level set method to track the users and guide them to the exit and the boundary of the hazardous areas. So, the people near to the dangerous areas will receive a mild congestion at the cost of slight detour. While, the people away from the dangerous areas will faces a unwanted detours. Generally, when an emergency situation occurs, people look for known path to the safe exit from their location.
\end{abstract}

Keywords: Wireless Sensor Network, GSTEB Protocol, CANS, Emergency Navigation algorithm.

\section{INTRODUCTION}

The developments in the wireless sensor network technologies provide the ability to use sensors in wide range of applications and in different fields of interests. But in earlier days, the sensors are designed to collect the data to monitor the physical changes in the environment. And later they are used to develop the in-situ interactions between people and their physical environment, which is used to significantly expand the usability and capability of wireless sensor networks. The major application of such in-situ interactions was WSN assisted emergency navigation, in which the WSN infrastructure is utilized as a cyber-physical system. In the present mobile environment, the mobile users equipped with smart phones or any electronic devices or PDA's that can talk with sensors. When any emergency situation occurs, the WSN creates the emergency field and provides the necessary guidance to the user. So that, the mobile users are guided to move out of a dangerous region with the ubiquitous interaction with sensors.

In the earlier years, one should have the thought of trivial usage of traditional packet routing protocols for guiding navigation. Moreover, there are some inbuilt features of emergency navigation that extensively differentiate itself from packet routing schemes. In packet routing schemes, the energy efficiency or data loss is considered as the first priority rather than a safe-critical path, which is a main aspect of human beings in emergency navigation. Here, the safety is not only refers to the far away from dangerous areas but, also refers to mild detours, less congestion and mainly fast reaction to the emergency situations. Secondly, the human navigation takes much more time than the traditional packet routing scheme, due to the partial movement speed of people. In the packet delivery process, the network is often considered as static, but the human navigation in contrast deals with the emergency dynamics which are all along the guiding process. Due to this, we cannot simply take the existing packet routing schemes for emergency navigation with WSNs. A variety of distinctively designed solutions for emergency navigation approach with WSNs has been proposed. With this approach we mainly focus on finding the shortest path to the safe location along with small stretch, mild congestion and fewer detours.

The major application of wireless sensor networks is the emergency service for the people who needs emergency evacuation. The navigation service is used in various domains such as industrial applications, military applications, and 
Vol. 6, Issue 6, June 2017

in environmental situations. The emergency responders need location information and navigation support to solve the environmental issues. The navigation application is mainly exists between sensors and the users. In this paper, we propose a CANS algorithm which is the first wireless sensor network assisted emergency navigation algorithm which achieves both less congestion and small stretch. The operations carried out in this are in-situ which is carried out by cyber-physical interactions between people and the sensor nodes. CANS use the technique of level set method to track the evolution of the exit and the boundaries of dangerous areas. So that, the people nearby the dangerous areas will achieve a less congestion and small stretch. The people away from the dangerous areas will receive an unnecessary detour. This algorithm CANS, do not require the location information and CANS algorithm contains level set methods. For this it establishes three methods. Namely, first method to establishing the potential map, the second method is used to building the hazard level map and final method is planning a safe path to each user. Note that in this potential map and hazard level map is combined to produce a compound level map. The main aim of the project is to guide people to escape from the dangerous areas quickly when an emergency situation occurs, with safety guarantee and with less congestions and small detours.

\section{Methodology}

\section{CANS Algorithm:}

CANS is a new algorithm developed by Wang in 2016 to overcome the problems of heavy congestions and detours during the fast movement of mobile users when an emergency situation occurs. And which means congestion adaptive small stretch and emergency navigation algorithm. The CANS algorithm, which is the first wireless sensor network that assist emergency navigation, which achieves both less blocking and minute stretch. The main work carried out in this algorithm is in-location or in same location, which is conceded by cyber-physical interactions between human beings and the mobile sensor nodes. The algorithm CANS use the technique of level set technique to follow the advancement of the outlet and the margins of dangerous areas. So, people close to the dangerous locationss will attain a less blocking and minute stretch. The people away from the dangerous areas will receive unnecessary detours. This algorithm CANS, do not require the location information and CANS algorithm contains level set methods. For this it establishes three methods. Namely, first method to establishing the potential map, the second method is used to building the hazard level map and final method is planning a safe path to each user. Note that in this potential map and hazard level map is combined to produce a compound level map. The main aim of the project is to guide people to escape from the dangerous areas quickly when an emergency situation occurs, with safety guarantee and with less congestions and small detours. The main advantage of CANS algorithm is that, it can be dispersed and impactiful to the size of the network, in which the nodes of the network has limited storage capability. It does not depend on any of the particular communication models.

\section{CANS Algorithm:}

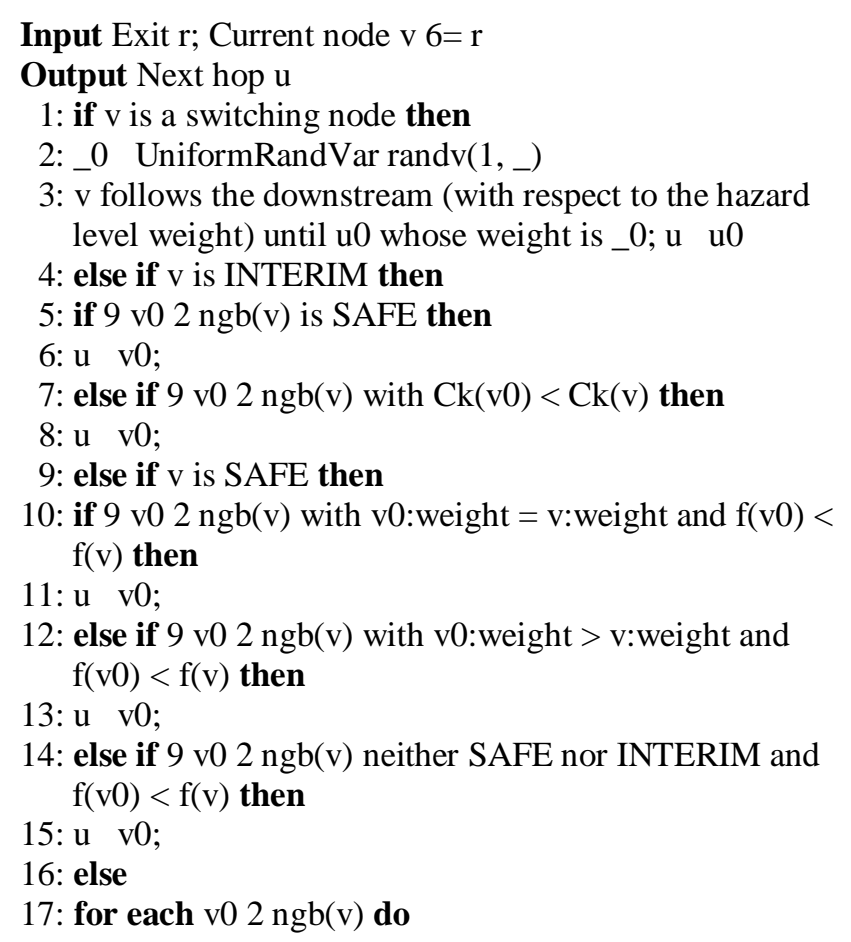


18: if $\mathrm{f}(\mathrm{v} 0)<\mathrm{f}(\mathrm{v})$ then

19: u v0; break

20: return $u$

By using CANS algorithm, we transport a level set perception and which does not look towards a topological construction that apprehends the large-scale attributes of the network. Apart from that, we mainly focus on confined regional anatomy, particularly in the region of the dangerous locations. Our basic initiative is mainly depend on the subsequent observations: such as mobile users extreme from the dangerous areas are frequently satisfactorily protected. So that they can try tolerant the more congestions, but the mobile users close to the risky areas are exposed to congestions. Based On this, we intend to build a compound map of the system, together with potential map and with hazard level maps are also constructed for emergency navigation. The potential map tracks the contrary development of level sets headed for the exit. And thus, it develops a sense of navigation path by which mobile users can safely headed for the exit with some detour. While the hazard level map tracks the growth of level sets off the harmful areas, and therefore, they can obviously advise where the dangerous areas are located, and further aid the mobile users close to the dangerous areas to be divided into different paths to evade serious congestions. By doing this, the mobile users can be guided to the exit in a reasonable safety way. The mobile users near by the dangerous areas accomplish a gentle congestion at the cost of a trivial detour. But, people away from the threat will evade avoidable detours.

\section{PROPOSED SYSTEM}

\section{Network Formation:}

In the present digital environment, the users in the network are equipped with electronic gadgets like cell phones, tabs, and any other PDA's which are capable of acting as sensors and establish the communication between the users and the network administrator. Here, the network means any public areas like complexes, railway stations, private malls, clubs, shopping complexes, airports etc. like these type of networks the users are act as mobile users and the communication establishes between them is by sending requests in between mobile users and the network administrator. The above case is the real situation which is mapped with nodes creation in the network simulator tool. If any emergency situation occurs in the network like bomb blasts, fire accidents or any other dangerous situations the users in the network escapes from the network to the safe location within a short time is serious problem. To overcome the above problem, an algorithm called CANS is developed by the wang in 2016. He maps the situation with the normal nodes in the network simulator. These are created in network animator (named nam window) by using the tcl script described in the NS2 installation process in the system requirements. In the nam window we create nearly 30 nodes to map the real public environment in the public places. And we deploy the connections between nodes for the communication between them. And we apply the duplex communication between nodes and also apply the connections like TCP, UDP, IP etc. and the networks like WAN, WLAN etc.

\section{CANS Architecture:}

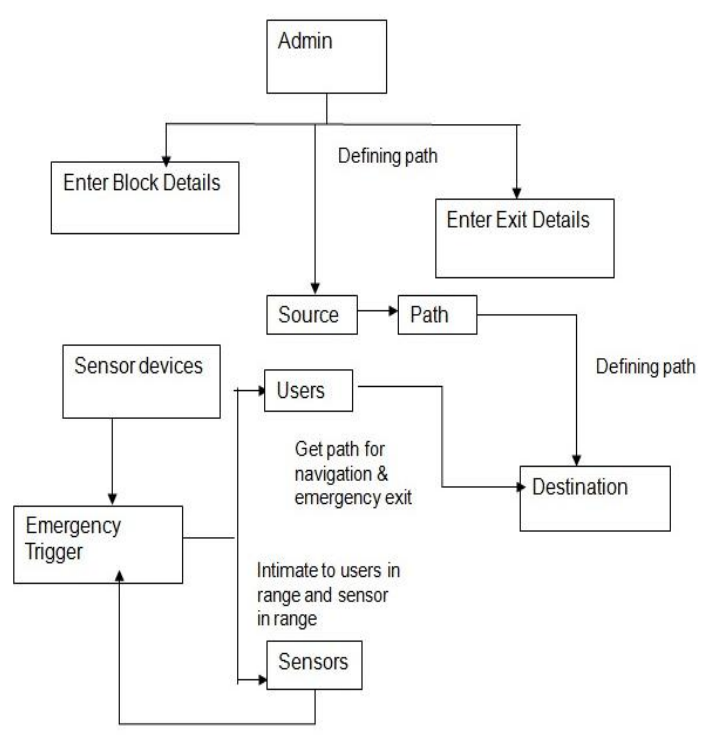

Fig-1:CANS Algorithm Architecture 
The above diagram shows the architecture of CANS algorithm. By using CANS algorithm, when any emergency situation occurs, the sensors in the wireless sensor networks take the information on overall condition of the surroundings and location information of the all the users. And then the sensors in the wireless sensor networks talk with the mobile users about the better and near way to the exit or safe path. CANS execute the technique of level set method to track the users and guide them to the exit and the boundary of the hazardous areas. So, the people near to the dangerous areas will receive a mild congestion at the cost of slight detour. While, the people away from the dangerous areas will faces a unwanted detours. Generally, when an emergency situation occurs, people look for known path to the safe exit from their location. They don't look for sub-optimal paths which takes the users to the safe location with less time from their location. CANS implementation using GSTEB protocol in wireless sensor network makes the use of these sub-optimal paths to the exit and thereby it decreases the congestion. By using the CANS algorithm, the users are guided to the safest locations when an emergency situation occurs with mild congestion and small detour.

\section{Congestion Avoidance Problem:}

In emergency situations, congestion is the main problem establish between the users when they seek for safest path. The human navigation is slightly differing from the traditional packet routing. Mainly, the human navigation will look for a safe path when an emergency occurs more than a energy efficiency or packet loss which are the mostly happening processes done in traditional packet routing. Other than that, when compared with the packet routing the human navigation takes more time to travel because of the limited speed of the human beings. Except CANS, the other algorithms will only seek for well known rout to exit. But, they never try to control the problem of congestion. By using CANS algorithm, wang try to rectify the problem of congestion.

But, he cannot rectify the problem completely. Because CANS doesn't any location information. Without any location information, guiding the users for safe path is practically impossible. So, we propose the GSTEB protocol to guide the people in dangerous situations for a safe exit. By using CANS algorithm, when any emergency situation occurs, the sensors in the wireless sensor networks take the information on overall condition of the surroundings and location information of the all the users. And then the sensors in the wireless sensor networks talk with the mobile users about the better and near way to the exit or safe path. CANS execute the technique of level set method to track the users and guide them to the exit and the boundary of the hazardous areas. So, the people near to the dangerous areas will receive a mild congestion at the cost of slight detour. While, the people away from the dangerous areas will faces a unwanted detours. Generally, when an emergency situation occurs, people look for known path to the safe exit from their location. They don't look for sub-optimal paths which takes the users to the safe location with less time from their location. CANS implementation using GSTEB protocol in wireless sensor network makes the use of these sub-optimal paths to the exit and thereby it decreases the congestion. By using the CANS algorithm, the users are guided to the safest locations when an emergency situation occurs with mild congestion and small detour.

\section{SIMULATIONS}

Low Energy Adaptive Clustering Hierarchy is an efficient routing protocol in wireless sensor networks. It is an Time domain multiple accessing (TDMA) based MAC protocol which is integrated with clustering and a normal routing protocol in wireless sensor networks. The main goal of LEACH protocol is to lower the energy consumption for creating the nodes and maintain the clusters to improve the lifetime of wireless sensor networks.

The General Self-Organized Tree-Based Energy-Balance Routing Protocol is a dynamic routing protocol for wireless sensor networks. For each cluster head node election process, the base station proposes a node for cluster head. And each node selects its cluster head based on its own and neighbouring nodes information. Which node has highest weight, in term of neighbouring nodes opinions will select as cluster head. Likewise, the cluster head formation occurs in the GSTEB protocol. It is mainly used to balance the energy consumption and thus, to prolong the lifetime of wireless sensor networks.

These two protocols are used here to implement the CANS algorithm. CANS implementation using LEACH protocol has done earlier. But, we implement the CANS now with GSTEB protocol. When we compare the CANS implementation with the both protocols the performance analysis of CANS is explained below with the following figures.

\section{Propagation Delay:}

Propagation delay is the amount of time taken by the signal to travel from the sender to the receiver. It can be computed as the ratio between the link length and the propagation speed over the specific medium. Propagation delay is equal to $\mathrm{d} / \mathrm{s}$ where $\mathrm{d}$ is the distance and $\mathrm{s}$ is the wave propagation speed. In wireless communication, $\mathrm{s}=\mathrm{c}$, i.e. the speed of light. In copper wire, the speed s generally ranges from $.59 \mathrm{c}$ to $.77 \mathrm{c}$. This delay 
Vol. 6, Issue 6, June 2017

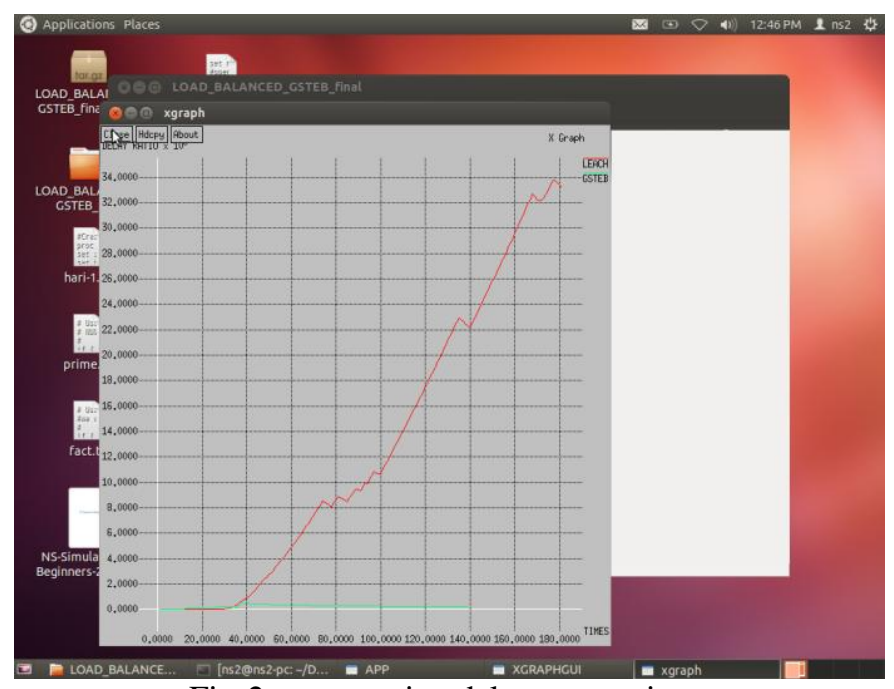

Fig-2: propagation delay comparison

Total loss: Total loss is defined as the total power consumed by the sensor networks to processs the communication between the nodes. The mobile users in the network are defined as nodes in the network. Generally, in present day environment the nodes in the wireless sensor network are mobiles, laptops, tabs, PDA's or any other digital electronic devices. These electronic devises are act as nodes to sense the physical conditions of environment and to transfer the request and responses from the base station.

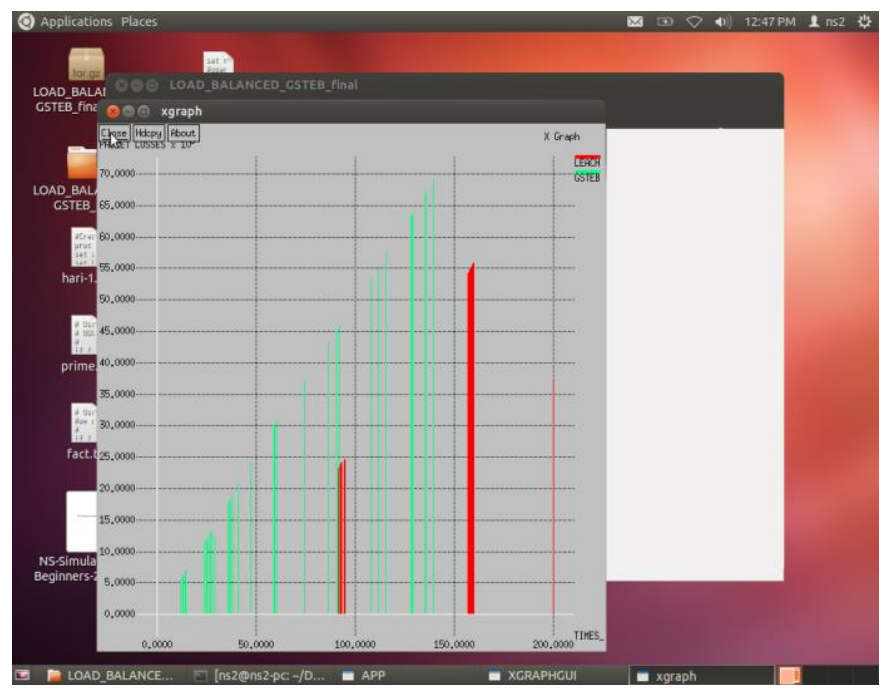

Fig-3: Total Loss Comparison

When comparing the total loss of both LEACH and GSTEB protocols the energy consumption is low in GSTEB protocol and is high in LEACH protocol. Because GSTEB is a dynamic routing protocol, which changes its cluster heads when it consumes more power. Unlike GSTEB, LEACH is a static routing protocol in which it doesn't change its cluster head when it consumes more power.

\section{Throughput workload:}

The throughput is a measure of calculating the how much amount of data transferred from source to destination and the workload is defined as the how much number of tasks performed in certain given time. In certain amount of time how much information transferred from source to destination is defined as throughput workload.

When comparing the throughput workload of both the protocols, the throughput workload of GSTEB is higher than the LEACH protocol. In GSTEB protocol throughput workload is high because it transmits the data very fastly. As the workload increases the transmitting time decreases in the other protocols, but in GSTEB protocol transmitting time remain same because it is a dynamic protocol and the communication between the nodes was accurate and transmits the data in with less time. 


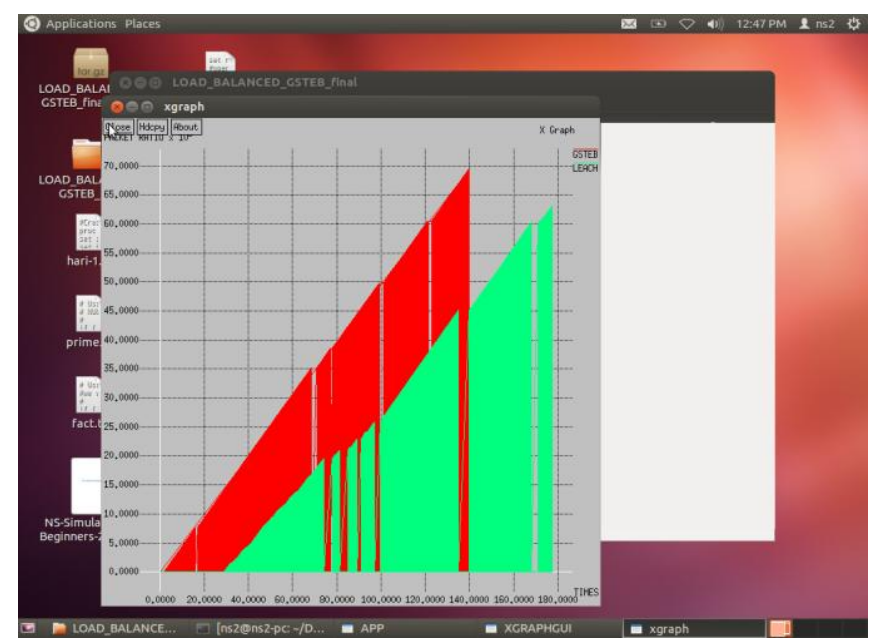

Fig-4: Throughput Workload Comparison

\section{Node-Node packet delivery:}

Node packet delivery is defined as the capability of nodes in the wireless sensor networks to deliver the packet or transmit the data. It mainly depending on the type of transceivers used in the users electronic equipments. The other properties like signal strength, propagating frequencies will change the node packet delivery of the network.

In the existence model, the node packet delivery is same for each node i.e. each node utilizes the same transceivers for transmission and reception. In the proposed model, the node packet delivery is varying and has huge response with large nodes and have highest packet delivery ratio when compared with existence system.

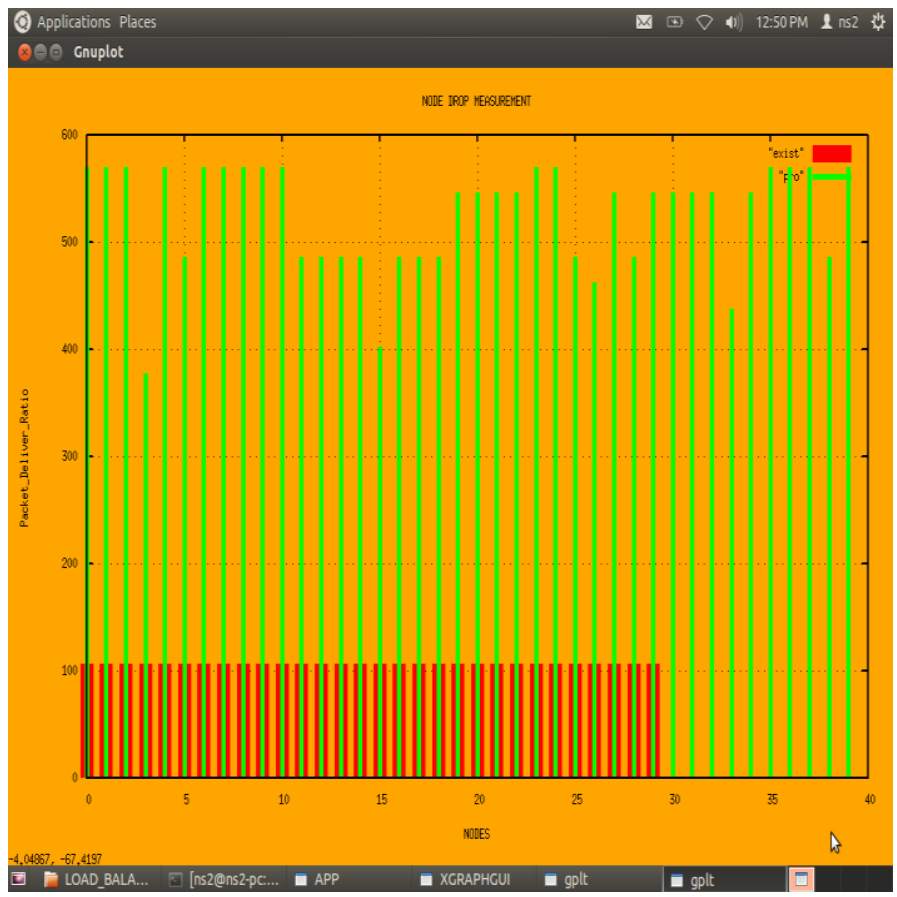

Fig-5: Node-Node Packet Delivery

\section{CONCLUSION}

In this project we present a noval algorithm named CANS algorithm to find the solutions of the problems raised during emergency navigation. This algorithm is already in use. But, it has some limitations like it doesn't need any location information from the previous protocols used to implement CANS algorithm. Now, in this we use a GSTEB protocol to provide safest path with in small time and with mild congestion and small detour by using level set method to track the location. In GSTEB protocol, the nodes cluster head formation was takes place dynamically, which makes the protocol differ and efficient from other traditional protocols. In this, we add extra feature called navigation service to the algorithm to make algorithm more effective to find the safe path in short time with less detours and small congestion. 


\section{IJARCCE}

Vol. 6, Issue 6, June 2017

\section{REFFERENCES}

[1] I. F. Akyildiz and M. C. Vuran, Wireless Sensor Networks. New York, NY, USA: Wiley, 2010.

[2] Y. Song, B. Wang, Z. Shi, K. Pattipati, and S. Gupta, "Distributed algorithms for energy-efficient even self-deployment in mobile sensor networks," IEEE Trans. Mobile Comput., vol. 13, no. 5, pp. 1035-1047, May 2014.

[3] C. Fischer and H. Gellersen, "Location and navigation support for emergency responders: A survey," IEEE Pervasive Comput., vol. 9, no. 1, pp. 38-47, Jan.-Mar. 2010.

[4] J. Wang, Z. Li, M. Li, Y. Liu, and Z. Yang, "Sensor network navigation without locations," IEEE Trans. Parallel Distrib. Syst., vol. 24, no. 7 , pp. 1436-1446, July 2013.

[5] M. Bocca, O. Kaltiokallio, N. Patwari, and S. Venkatasubramanian, "Multiple target tracking with RF sensor networks," IEEE Trans. Mobile Comput., vol. 13, no. 8, pp. 1787-1800, Aug. 2014.

[6] Q. Li, M. De Rosa, and D. Rus, "Distributed algorithms for guiding navigation across a sensor network," in Proc. 9th Annu. Int. Conf. Mobile Comput. Netw., 2003, pp. 313-325.

[7] E. Xu, Z. Ding, and S. Dasgupta, "Target tracking and mobile sensor navigation in wireless sensor networks," IEEE Trans. Mobile Comput., vol. 12 , no. 1, pp. 177-186, Jan. 2013

[8] C. Buragohain, D. Agrawal, and S. Suri, "Distributed navigation algorithms for sensor networks," in Proc. 25th IEEE Int. Conf. Comput. Commun., 2006, pp. 1-10.

[9] Y.-C. Tseng, M.-S. Pan, and Y.-Y. Tsai, “Wireless sensor networks for emergency navigation,” Computer, vol. 39, no. 7, pp. 55-62, July 2006.

[10] Q. Li and D. Rus, "Navigation protocols in sensor networks," ACM Trans. Sensor Netw., vol. 1, no. 1, pp. 3-35, 2005. 